Pramāṇa -J. Phys., Vol. 25, No. 4, October 1985, pp. 467-472. (C) Printed in India.

\title{
Bose condensation of particle-antiparticle systems
}

\author{
K P SINHA \\ Indian Institute of Science, Bangalore 560012, India
}

\begin{abstract}
We discuss some important papers that have appeared in the last twenty years on the possibility of Bose condensation in particle-antiparticle systems. Electron-hole systems in some semiconductors provide the background for a non-relativistic treatment. Bose condensation and the superfluid phase of the electron-hole fluid are strongly favoured. Next, pairing and the appearance of the superfluid vacuum state in fermion-antifermion system are considered from a relativistic viewpoint. Special attention is given to the pairs in the state $J^{P}=0^{+}$. The pairing in the fundamental fermion-antifermion sea may provide the background subquantal level of reality of the universe.
\end{abstract}

Keywords. Bose condensation; particle-antiparticle system; electron-hole system; fermionantifermion sea.

\section{Introduction}

In the present paper we discuss some important developments that have taken place in the last twenty years concerning Bose condensation in particle-antiparticle systems. First we consider a non-relativistic situation which occurs in a solid, namely interacting electron-hole system in a semiconductor. Next, we discuss the relativistic treatment of particle-antiparticle pairing. This is important in the context of the nature of the vacuum state in field theory and the subquantal level of reality of the universe (Sinha $e t$ al 1976; Vigier 1980).

In solids the system comprises conduction $(c)$ band electrons and valence $(v)$ band holes. These electron-hole systems can be produced in solids where $c$ and $v$ bands overlap (indirect gap) or generated by optical pumping (direct band gap systems). The former has been the subject of study of electron-hole $(e-h)$ pairing leading to excitonic insulators and the latter to electron-hole droplet condensation (actually seen experimentally) and the possibility of Bose condensation and superfluidity (Kjeldysh and Kopaev 1965; des Cloizeaux 1965; Jerome et al 1967; Comte and Nozieres 1982; Nozieres and Comte 1982).

\section{Electron-hole system in solids}

Consider a single pair of $e-h$ system in a direct gap semiconductor with $m_{e}=m_{h}$. They form a bound state (exciton) of energy $-\varepsilon_{0}$ and radius $a_{0}$. In the regime $m_{e}=m_{h}$ formation of biexcitons can be ignored. Let us define the number of electrons $\left(N_{e}\right)$ and holes $\left(N_{h}\right)$, which satisfy $N_{e}=N_{h}=N$. One has to examine the density regimes of excitons from $\mathrm{Na}_{0}^{3} \ll 1$ (dilute) to $\mathrm{Na}_{0}^{3} \gg 1$ (dense) where excitons overlap and one must 
take account of the two fermions that constitute the bosons (Comte and Nozieres 1982). Ignoring spin for a while, the exciton creation operator can be defined as

$$
\psi_{\mathrm{ex}}^{+}=\Sigma \phi_{k} a_{k}^{+} b_{k}
$$

where $a_{k}^{+}$is the electron creation operator in the $c$ band and $b_{k}^{+}$for the $v$ band. A Kjeldysh type wave function for $N$ Bose-condensed excitons or electron-hole pairs is given by

$$
\begin{aligned}
\left|\Phi_{\lambda}^{0}\right\rangle & =\exp \left(\lambda \psi_{\mathrm{ex}}^{+}\right)|\mathrm{vac}\rangle \\
& =\prod_{k} \exp \left(\lambda \phi_{k} a_{k}^{+} b_{k}\right)|\mathrm{vac}\rangle \\
& =\prod_{k}\left(1+\lambda \phi_{k} a_{k}^{+} b_{k}\right)|\mathrm{vac}\rangle .
\end{aligned}
$$

This is a special case of Bardeen-Cooper-Schrieffer (BCS 1957) like ansatz

$$
\begin{aligned}
& \left|\Phi_{\lambda}^{0}\right\rangle=\prod_{k}\left[u_{k}+v_{k} a_{k}^{+} b_{k}\right]|\mathrm{vac}\rangle, \\
& u_{k}=\frac{1}{\left(1+\left|\lambda \phi_{k}\right|^{2}\right)^{1 / 2}}, \quad v_{k}=\frac{\lambda \phi_{k}}{\left(1+\left|\lambda \phi_{k}\right|^{2}\right)^{1 / 2}} .
\end{aligned}
$$

For a dilute exciton gas $u_{k} \sim 1$ and $v_{k} \simeq \lambda \phi_{k} \ll 1$. With increasing $N$ (pair density) $\left|v_{k}\right|^{2}$ increases and is stopped at the value 1 . This is a reflection of the normalization condition $u_{k}^{2}+v_{k}^{2}=1$ and in effect takes care of the exclusion principle in that electrons and holes are fermions. Physically this implies that the $N$ exciton states exhaust the underlying fermion states leading to saturation of $v_{k}$ when $\mathrm{Na}_{0}^{3} \sim 1$ and excitons start overlapping.

In the dense limit also $\left(\mathrm{Na}_{0}^{3} \gg 1\right)$, the BCS ansatz adequately describes the degenerate electron-hole plasma. Now we have taken $\left|v_{k}\right|^{2}$ as a step function

$$
\left|v_{k}\right|^{2}= \begin{cases}1 & \text { if } k<k_{F} \\ 0 & \text { if } k>k_{F}\end{cases}
$$

where $k_{F}=\left(6 \pi^{2} N\right)^{1 / 3}$ (ignoring spin). Thus in an isotropic $e-h$ plasma we get $e-h$ pairing analogous to BCS pairing in electron fluid. We have Bose condensation at all densitiesdilute exciton gas-excitonic insulator-e-h plasma.

For computing the gap function one can use BCs type extended meanfield approximation with $\left\langle a^{+} b\right\rangle$ anomalous pairing and $\left\langle a^{+} a\right\rangle,\left\langle b b^{+}\right\rangle$, Hartree-Fock pairing. Explicit solution is possible in the high density $\mathrm{Na}_{0}^{3} \gg 1$ limit. For unscreened coulomb interaction the gap turns out to be (Comte and Nozieres 1982).

$$
\Delta=E_{\mathrm{c}} \exp \left[-1 /\left(4 \pi e^{2} N_{0} / 3 k_{F}^{2}\right)^{1 / 2}\right]
$$

where $E_{c}$ is the energy cut-off of the order of Fermi energy $E_{F}$ and. $N_{0}$ the density of states at the Fermi level. This shows that the gap vanishes exponentially when $N \rightarrow\left(k_{F}\right.$ $\left.=\left(6 \pi^{2} N\right)^{1 / 3}\right)$. Thus $\Delta$ for $N \rightarrow 0$ starts from a vanishing value and passes through a maximum at some critical value $N_{c}$ and then falls off.

The ground state possesses collective excitations, bound quasiparticle pairs or fluctuations of the order parameters $\left\langle a^{+} b\right\rangle$. In the dilute limit excitations are phonons and rotons of the Bose system and in the dense limit it will sustain Bogoliubov modes in the gap. Quantitative calculation of the critical temperature $T_{c}$ at which Bose 
condensation disappears is not possible as the Kjeldysh function does not allow a smooth description of $T_{c}$ through the whole density range. Qualitatively, for large $N, T_{c}$ is exponentially small and of the order of $\Delta$. In the limit $N \rightarrow 0, T_{c} \sim N^{2 / 3}$. In between $T_{c}$ should go through a maximum, which is a pure conjecture.

A spin-dependent formulation of the electron-hole system inclusive of screening and band structure effects have been considered by Nozieres and Comte (1982). For spin 1/2 particles the wave function can be written as

$$
\left|\Psi_{0}\right\rangle=\exp \left[\Sigma \lambda_{\sigma \sigma^{\prime}} \phi_{k} a_{k \sigma}^{+} b_{k \sigma^{\prime}}\right]|\mathrm{vac}\rangle
$$

where $\lambda$ is a $2 \times 2$ complex matrix and fixes the spin state. The choice

$$
\lambda=\left(\begin{array}{ll}
1 & 0 \\
0 & 1
\end{array}\right) \text { (singlet) and } \lambda=\left(\begin{array}{rr}
1 & 0 \\
0 & -1
\end{array}\right) \text { (triplet } S_{z}=0 \text { ) }
$$

corresponds to factorization of the ground state wavefunction

$$
\left|\Psi_{0}\right\rangle=A_{\uparrow} A_{\downarrow}|\mathrm{vac}\rangle \text {. }
$$

In this representation $\uparrow \uparrow$ and $\downarrow \downarrow$ pairs condense separately, with decoupled order parameters. The ground state energy is a sum $E_{0 \dagger}+E_{0 \downarrow}$ of non-interacting mixture. In a fully polarized state electrons and holes each have single spin states characterized by two directions $n_{e}$ and $n_{h}$. If they are parallel, the total state is triplet, otherwise we have a mixture. For arbitrary polarization, one will have $N_{1}$ and $N_{2}$ independent groups. Under mean field approximation, the ground state energy

$$
E_{0}=N_{1} E\left(V / N_{1}\right)+N_{2} E\left(V / N_{2}\right)
$$

In fact, spin polarization of excitons is equivalent to liquid-gas separation. Qualitatively, the energy as a function of density of the various spin states is as follows: In the dilute limit triplets $T_{0}$ and $T_{1}$ are close together and well separated from singlet $S$. In the high density $S$ and $T_{0}$ are very close and $T_{1}$ lies higher up.

Screening corrections in the framework of random phase approximations lead to an approximate form of Van der Waal attraction between excitons. Calculations are possible only in the dilute and dense regimes. For the latter the polarizability behaviour is the same as a normal plasma.

Intermediate density region is formidable and one can at best make primitive conjectures. Complications in band structure go against the Bose condensation. If the degeneracies of conduction and valence bands are different, we will have a normal plasma state. Thus the fermi surfaces must match and the degeneracies of the two bands should be the same for Bose condensation.

Instead of pairing of electrons and holes, one can have pairing of excitons also if an attractive interaction between these exists and excitons are fairly longlived. Excitonphonon can provide the necessary attractive interaction. The equation for the gap parameter $\Delta_{k}$ turns out to be (Nandakumaran and Sinha 1975)

$$
\Delta_{k}=\sum_{k^{\prime}} V_{\mathrm{ex}}\left(k-k^{\prime}\right) \frac{(1-2 n)}{2 E_{k^{\prime}}} \Delta_{k^{\prime}} \operatorname{coth}\left[\frac{\beta(1-2 n) E_{k^{\prime}}}{2}\right]
$$

where $n$ is the exciton density, $V_{\text {ex }}\left(k-k^{\prime}\right)$ the effective interaction between excitons and $E_{k}^{2}=\varepsilon_{k}^{2}-\left|\Delta_{k}\right|^{2}, \varepsilon_{k}$ being the exciton energy relative to its chemical potential. The 
excitation has the form

$$
\omega=(1-2 n)\left[T_{k}\left(T_{k}+2\left|\Delta_{k}\right|\right)\right]^{1 / 2},
$$

where $T_{k}$ is a redefined kinetic energy. Since $T_{k} \rightarrow 0$ as $k \rightarrow 0$, we get a phonon-like spectrum for small values of $k$ which signifies a superfluid phase for the excitons.

Recently Vignale and Singwi (1985) have explored the possibility of superconductivity in electron-hole liquid. The mediating bosons responsible for pairing of the electrons are correlated pair excitations from the Fermi sea of holes. They estimate $T_{c} \sim 1 K$.

\section{Fermion-antifermion sea}

Let us consider now superfluidity in a sea of fundamental fermions and anti-fermions. The Hamiltonian of this fundamental "sea" (Sinha et al 1976; Sinha and Sudarshan 1978 ) is given by

$$
\begin{aligned}
\mathscr{H}= & \Sigma \varepsilon_{k} c_{k \sigma_{-}}^{+} c_{k \sigma_{-}}+\Sigma \varepsilon_{k} d_{k \sigma_{+}}^{+} d_{k \sigma_{+}} \\
& -\Sigma g_{r}\left(k, k^{\prime}\right) d_{k^{\prime} \sigma_{-}}^{+} d_{q-k^{\prime}, \sigma_{+}}^{+} d_{q-k, \sigma_{+}} c_{k \sigma_{-}},
\end{aligned}
$$

where $\sigma_{-}$denotes the spin of fermion, $\sigma_{+}$that of anti-fermion and we have taken $\varepsilon_{k}^{(c)}$ $=\varepsilon_{k}^{(d)}=\varepsilon_{k}$ for single particle energy; $g_{r}\left(k, k^{\prime}\right)$ is the fundamental interaction between fermions and anti-fermions (creation operators $c_{k \sigma_{-}}^{+}, d_{k \sigma_{+}}^{+}$, etc respectively). For singlet spin pair we will have $S\left(=1 / 2\left(\sigma_{+}+\sigma_{-}\right)\right)$zero and for triplet unity. Also for the orbital states of the pairs the allowed angular momentum values are $L=0,1,2$, etc. Accordingly the various allowed states for the pair are $1 S_{0}, 3 S_{1}, 1 P_{1}, 3 P_{0}$, etc. For singlet pairing the quasiparticle energy $E_{k}$ turns out to be

$$
E_{k}^{2}=\varepsilon_{k}^{2}+\left|\Delta^{(0)}\right|^{2}
$$

and for triplet with all the three components equally populated, the isotropic energy spectrum is

$$
E_{k}^{2}=\varepsilon_{k}^{2}+\left|\Delta^{(1)}\right|^{2},
$$

where $\Delta^{(i)}$ 's are the two by two gap matrices. On the face, these results look similar to non-relativistic BCs type theory. However it can be shown that a relativistic treatment gives almost identical expressions. We follow the method described by Bailin and Love (1984). Let $\psi(x)$ be a relativistic fermion field with $\psi_{c}(x)$ its charge conjugate field. The free spinor field is described by the Lagrangian

$$
\mathscr{L}_{\text {free }}=\bar{\psi}\left(i \gamma^{v} \partial_{v}-m_{0}\right) \psi+\mu \bar{\psi} \gamma^{0} \psi,
$$

where $m_{0}$ is the bare mass and $\mu$ the chemical potential at finite density; $\gamma$ 's are the usual Dirac matrices. For present purposes, we ignore internal degrees of freedom (e.g., isospin, colour, etc). Then for a pair of fermion and antifermion in state $J^{P}=0^{+}$, the gap matrix will have the explicit form

$$
\left.\Delta_{(\mathbf{n})}^{(0)}\right)^{+}=\Delta_{1} \gamma_{5}+\Delta_{2} \mathbf{n} \cdot \gamma \gamma_{0} \gamma_{5}+\Delta_{3} \gamma_{0} \gamma_{5}
$$

We have considered a homogeneous superfluid and thus the gap matrix in momentum space depends on a single momentum variable. The form selected above depends only 
on the direction $\mathbf{n}$ (unit vector) and not on the magnitude of wave vector. The components $\Delta_{1}, \Delta_{2}$ and $\Delta_{3}$ depend on the models taken for exchange producing pairing. It is possible to obtain a model independent form by taking a trace to project out.

$$
\Delta^{(0)}=\Delta_{1}-\frac{p_{F}}{\mu} \Delta_{2}-\frac{m_{0}}{\mu} \Delta_{3}
$$

where $p_{F}$ is the Fermi momentum. One gets the BCs-like integral equation for gap

$$
\Delta^{(0)}=\frac{1}{4} N(\varepsilon) a_{c}^{-1} \Delta^{(0)} \int_{-\infty}^{\varepsilon_{0}} \mathrm{~d} \varepsilon\left(\varepsilon^{2}+\Delta^{(0) *} \Delta^{(0)}\right)^{-1 / 2} \tanh \frac{\beta}{2}\left(\varepsilon^{2}+\Delta^{(0) *} \Delta^{(0)}\right)^{1 / 2}
$$

where $\varepsilon_{0}$ is the energy cut-off and $N(\varepsilon)$ is the density of states and

$$
\begin{aligned}
a_{c}^{-1}= & \frac{1}{16} g^{2} \int \frac{\mathrm{d} \Omega}{4 \pi} \int \frac{\mathrm{d} \Omega^{\prime}}{4 \pi} D_{A B}\left(\mathbf{n}, \mathbf{n}^{\prime}\right) \\
& \times T_{r}\left\{\left(\gamma_{5}+\frac{p_{F}}{\mu} \mathbf{n}^{\prime} \cdot \gamma \gamma_{0} \gamma_{5}+\frac{m_{0}}{\mu}\right) \tilde{\Gamma}^{A}\right. \\
& \left.\times\left(\gamma_{5}+\frac{p_{F}}{\mu} \mathbf{n} \cdot \gamma \gamma_{0} \gamma_{5}-\frac{m_{0}}{\mu}\right) \Gamma^{B}\right\},
\end{aligned}
$$

where $D_{A B}$ is the propagator for the exchanged boson, (ig $\left.\Gamma^{A}\right)$ appears at the interaction vertex; $\Gamma^{A}$ takes care of internal symmetry if any. Note that integration is over angle variables $\Omega$ and $\Omega^{\prime}$.

Equation (17) is solved at $T=0$, i.e. to give the gap as

$$
\left|\Delta^{(0)}\right|_{T=0}=2 \varepsilon_{0} \exp \left[-\left(\frac{1}{2} N(\varepsilon) a_{c}^{-1}\right)^{-1}\right] .
$$

The critical temperature $T_{c}$ is obtained for $\left|\Delta^{(0)}\right| \rightarrow 0$. One finds

$$
T_{c}=\frac{1 \cdot 14 \varepsilon_{0}}{k_{B}} \exp \left[-\left(\frac{1}{2} N(\varepsilon) a_{c}^{-1}\right)^{-1}\right] .
$$

Both (19) and (20) have the same structure as the non-relativistic BCS case. The superfluid sea of fundamental fermion-antifermion fields forms the background (non-empty) vacuum of the observable universe. Various Bose-like excitations, scalars, vectors and tensors are composites of these fundamental matter and become the vehicles of observed forces in nature. These fundamental fermions also lead to other composites, nucleons, atoms, etc and hence the rest of the material world. The pair $\mathrm{J}^{P}=0^{+}$, may be identified with Higgs-like particles. Finally, even the geometry (the metric describing space-time) may be built out of this fundamental fermionic matter (Akama 1978). This scheme is called "pregeometry"-the vacuum of the fundamental matter (preonic matter!).

Considerable work is still needed before one can have a unified picture of space, time and matter. 